\title{
DOENÇA DE CHAGAS: PERFIL DE MORBIDADE HOSPITALAR NA REGIÃO DO NORDESTE BRASILEIRO
}

\author{
Alison Pontes da Silval \\ Francisco Patrício de Andrade Júnior " \\ Bruna Braga Dantas II
}

\begin{abstract}
RESUMO
A Doença de Chagas é uma infecção parasitária que acomete milhões de indivíduos no mundo, principalmente, os que vivem em países marcados pela pobreza. Assim, o presente estudo tem por objetivo avaliar variáveis associadas a morbidade hospitalar por Doença de Chagas na região Nordeste do Brasil. Para isso, foram utilizados os dados armazenados no Sistema de Informações Hospitalares do SUS, referentes ao número de internações e de óbitos, taxa de mortalidade hospitalar, valor médio por internação e valor total das internações, regime, caráter de atendimento, sexo e cor/raça, considerando a população do Nordeste acometida pela Doença de Chagas, no período de 2008 a 2018. Os dados obtidos apontaram que o ano de 2008 deteve os maiores registros em internações e em gastos com casos clínicos de Doença de Chagas, apesar deste mesmo ano apresentar a menor taxa de mortalidade em todo o período. Além disso, de 2008 a 2018 foram gastos US\$1.272.109,80 em internações, o que equivale a um valor médio por internação de US\$ 675,20. A maior parte das internações foram realizadas no sistema público de saúde, além de serem de caráter de urgência. Também se verificou que houve predominância de indivíduos do sexo masculino, de cor parda e com 60 anos ou mais de idade. Desse modo, pode-se perceber que houve uma diminuição considerável do número de internações hospitalares, ao longo do período estudado, bem como nos gastos associados as internações, o que divergiu com o aumento na mortalidade que não apresentou nenhuma alteração linear ao longo dos anos.
\end{abstract}

PALAVRAS-CHAVE: Tripanossomíase Americana. Hospitalização. Serviços Hospitalares. Sistemas de Informação Hospitalar.

Acadêmico do curso de Farmácia. Departamento de Farmácia. Universidade Federal de Campina Grande - UFCG. CEP: 58175000, Cuité, Paraíba, Brasil. ORCID/ID: https://orcid.org/0000-0001-8807-1601

Bacharel em Farmácia. Mestrando em Produtos Naturais e Sintéticos Bioativos, Universidade Federal da Paraíba, Centro de Ciências da Saúde, CEP: 58033-455, João Pessoa, Paraíba. Brasil. ORCID/ID: https://orcid.org/0000-0002-9662-4111

Enfermeira, Doutora em Biotecnologia, Centro de Educação em Saúde - Universidade Federal de Campina Grande,UFCG. CEP: 58175000, Cuité, Paraíba, Brasil.Email: brunabdantas@gmail.com. ORCID/ID: https://orcid.org/0000-0002-9662-4111 


\section{INTRODUÇÃO}

As doenças negligenciadas apresentam uma série de características em comum, como o fato de estarem marcadamente relacionadas a condições de pobreza, bem como a escassez de opções terapêuticas seguras e eficazes. Dentro desse grupo, destaca-se a Doença de Chagas, também conhecida como Tripanossomíase americana, uma parasitose endêmica em países do continente americano. A Organização Mundial da Saúde estima que 8 milhões de pessoas estejam infectadas, 10 mil pessoas morram por ano em decorrência de manifestações clínicas da doença e mais de 25 milhões estejam vulneráveis a adquirir a doença no mundo todo. ${ }^{1,2}$

A descoberta da Doença de Chagas ocorreu em 1909, pelo médico brasileiro Carlos Chagas. O agente etiológico desta doença parasitária é o protozoário Trypanosoma cruzi que pode infectar o ser humano por diversas vias, como fezes infectadas de triatomíneos (conhecidos popularmente no Brasil como barbeiros), transfusão sanguínea, transplante de órgãos, transplacentária, ingestão de alimentos contaminados e por acidente de trabalho. ${ }^{3}$

Após a infecção, a progressão da doença pode ser dividida em três fases: aguda, crônica assintomática e crônica sintomática. A fase aguda pode ser caracterizada por elevada parasitemia e pela ausência de sintomas em $90 \%$ dos casos, podendo ocorrer na porcentagem remanescente, febre, mal estar, surgimento de um nódulo na região ocular (sinal de Romanã) e/ou cutâneo (chagoma de inoculação), entre outros. É importante pontuar que a fase aguda com a presença de sintomas pode ser mais comum e severa em crianças, uma vez que podem ocorrer complicações como miocardite e meningoencefalite, as quais aumentam as chances de óbito. A fase crônica assintomática, por sua vez, é marcada por sorologia e/ou parasitemia positiva. Em 70-80\% dos casos, há uma persistência desta forma clínica, enquanto que nos demais há uma evolução para a fase crônica sintomática, após alguns anos ou décadas. Nesa última, os níveis de parasitemia são oscilantes, sendo marcada por uma série de manifestações gastrintestinais e/ou cardíacas, resultando em comprometimento de funções vitais, que resulta em aumento do número de internações e óbitos. ${ }^{3-5}$

Estimativas recentes apontam um quadro preocupante no cenário brasileiro, visto que o número de indivíduos infectados pelo $T$. cruzi nas cinco regiões do país é de aproximadamente 2 milhões. 6

A região Nordeste do Brasil, mais especificamente, é composta por nove estados: Alagoas, Bahia, Ceará, Maranhão, Paraíba, Pernambuco, Piauí, Rio Grande do Norte e Sergipe. Possui uma extensão territorial de $1.544 .257 \mathrm{Km} 2$, ocupando a terceira colocação neste aspecto. A população desta região, no último censo, foi equivalente a 53.081.950 habitantes, o que corresponde a aproximadamente $30 \%$ da população residente do país. Além disso, a densidade demográfica do Nordeste equivale a 34,1 habitantes $/ \mathrm{km} 2$, sendo que há um predomínio da população urbana (73\%) em relação à rural (27\%). Destacase, ainda, que esta localidade é uma das mais pobres de todo o país, apresentando atividades econômicas majoritariamente associadas a agricultura, turismo, extratisvismo, entre outras $^{7}$ e que não se observam estudos epidemiológicos relacionados a morbidade hospitalar por Doença de Chagas necessitandose, assim, pesquisas relacionadas a esta temática.

Assim, o presente estudo tem por objetivo avaliar variáveis associadas a morbidade hospitalar por Doenças de Chagas na região Nordeste do Brasil, de 2008 a 2018. 


\section{MATERIAL E MÉTODOS}

O presente trabalho é do tipo documental-retrospectivo-analítico, em que foram utilizados os dados presentes no Sistema de Informações Hospitalares do SUS (SIH/SUS) da base de dados do DATASUS/Ministério da Saúde.

A coleta dos dados ocorreu entre maio e junho de 2019, considerando a população do Nordeste, durante o período de 2008 a 2018, sendo estes anos referentes ao período de processamento dos dados. As informações coletadas sobre Doença de Chagas utilizaram a Classificação Internacional de Doenças e Problemas Relacionados à Saúde - CID-10:a B57, correlacionadas ao local de internação, segundo as seguintes variáveis: internações, óbitos, valores totais e médios por internação, caráter e regime de internação, sexo, cor/raça e taxa de mortalidade hospitalar. Sendo a taxa ajustada calculada da seguinte forma:

\section{RESULTADOS E DISCUSSÃO}

A investigação da morbidade por Doença de Chagas na região Nordeste mostrou que o maior registro de internações $(n=374)$ ocorreu no ano de 2008, o que contrasta com o valor da taxa de mortalidade hospitalar (4,81\%) deste ano uma vez que essa foi a menor em todo o período analisado. Isso reflete uma baixa quantidade de óbitos ( $n=18$ ), em termos relativos, em que a maior parte das pessoas infectadas permaneceram internadas sem agravos letais, ou receberam alta hospitalar (Tabela 1).

Por outro lado, em 2017, observa-se uma redução significativa nas internações ( $n=149)$ e um número de óbitos elevado $(n=22)$, quando comparados a 2008, apresentando
Taxa de mortalidade hospitalar $=\left(\frac{\text { óbitos }}{\text { internações }}\right) \times 100$

Cabe pontuar que foi realizada a conversão dos valores referentes a custos em reais para dólares americanos, conforme a cotação do dia 09 de maio de 2019, em que 1 dólar era equivalente a três reais e oitenta e oito centavos.

Os dados coletados foram transferidos para o programa Microsoft Office ${ }^{\circledR}$ Excel 2013, o qual possibilitou a elaboração de gráficos e tabelas. A análise estatística foi realizada por meio do Software Statistical Package for Social Science (SPSS) versão 13.0, em que houve o cálculo das frequências absoluta e relativa, além da aplicação do teste Qui-Quadrado de Independência, em que $\mathrm{p}<0,05$ foi considerado estatisticamente significativo para a rejeição das hipóteses de nulidade. um expressivo registro de taxa de mortalidade hospitalar por Doença de Chagas $(14,77 \%)$, sendo considerada a maior taxa de mortalidade do período estudado. Quanto a taxa de mortalidade hospitalar por Doença de Chagas também contou com elevado registro $(8,74 \%)$ no ano de 2011 , quando comparado aos mesmos dados de 2008, uma vez que apresentou ainda os menores registros em internações ( $n=103)$ e óbitos ( $n=9)$ (Tabela 1). É importante ressaltar que o diagnóstico tardio é um fator que contribui para o aumento da mortalidade, sendo fundamental a detecção precoce da doença e a realização do tratamento de forma correta. 8 
TABELA 1: Internações, óbitos e taxa de mortalidade hospitalar associado a Doença de Chagas no Nordeste brasileiro, considerando o período de 2008 a 2018

\begin{tabular}{cccc}
\hline $\begin{array}{c}\text { Ano de } \\
\text { processamento }\end{array}$ & Internações & Óbitos & $\begin{array}{c}\text { Taxa de mortalidade } \\
\text { hospitalar (\%) }\end{array}$ \\
\hline 2008 & 374 & 18 & 4,81 \\
2009 & 177 & 21 & 11,86 \\
2010 & 157 & 9 & 5,73 \\
2011 & 103 & 9 & 8,74 \\
2012 & 109 & 13 & 11,93 \\
2013 & 121 & 14 & 11,57 \\
2014 & 138 & 18 & 11,04 \\
2015 & 176 & 24 & 13,64 \\
2016 & 158 & 13 & 8,23 \\
2017 & 149 & 22 & 14,77 \\
2018 & 222 & 18 & 8,11 \\
\hline \hline Total & 1884 & 179 & 9,5 \\
\hline \hline
\end{tabular}

Além disso, a diminuição do número de internações está intimamente relacionada com as iniciativas de controle da doença, visto que estudos realizados em camundongos indicam que reinfecções podem aumentar a severidade de lesões cardíacas em indivíduos no estado crônico da doença. 9

Considerando os registros hospitalares que caracterizam todo o período estudado, observa-se 1884 internações e 179 óbitos por Doença de Chagas, o que implica que a taxa de mortalidade hospitalar no período em estudo foi equivalente a 9,5\% (Tabela 1). Tal valor é semelhante ao relatado em um estudo sobre morbidade e mortalidade hospitalar referente à Doença de Chagas no Brasil, o qual obteve uma taxa de $9,05 \%{ }^{10}$ Isto permite inferir que apesar das peculiaridades da região do Nordeste, as características de morbidade hospitalar permancem em consonância com o Brasil.

Quanto a análise dos valores totais gastos em internações por Doença de Chagas no Nordeste, pode-se verificar que durante os anos de 2008 e 2011, houve o maior (US\$ $432.355,2$ ) e o menor (US\$ 56.865,9) registro de gastos, respectivamente, o que pode estar relacionado com o fato destes anos expressarem o valor mais alto e o mais baixo em internações, respectivamente. $O$ gasto total em internações por Doença de Chagas no período estudado foi de US\$1.272.109,80 (Figura 1). Uma análise dos custos por Doença de Chagas em todo o mundo apontou que esta parasitose é menos dispendiosa do que algumas doenças crônica de elevada incidência, como o câncer de mama, mas contém custos superiores a outras doenças infecciosas, a exemplo da cólera. ${ }^{11}$

Quando comparado a outras parasitoses, os relatos reforçam que impactos causados pela Doença de Chagas podem gerar custos, referentes a anos de vida ajustados por incapacidade, até 7,5 vezes maior que a malária. ${ }^{3}$ 


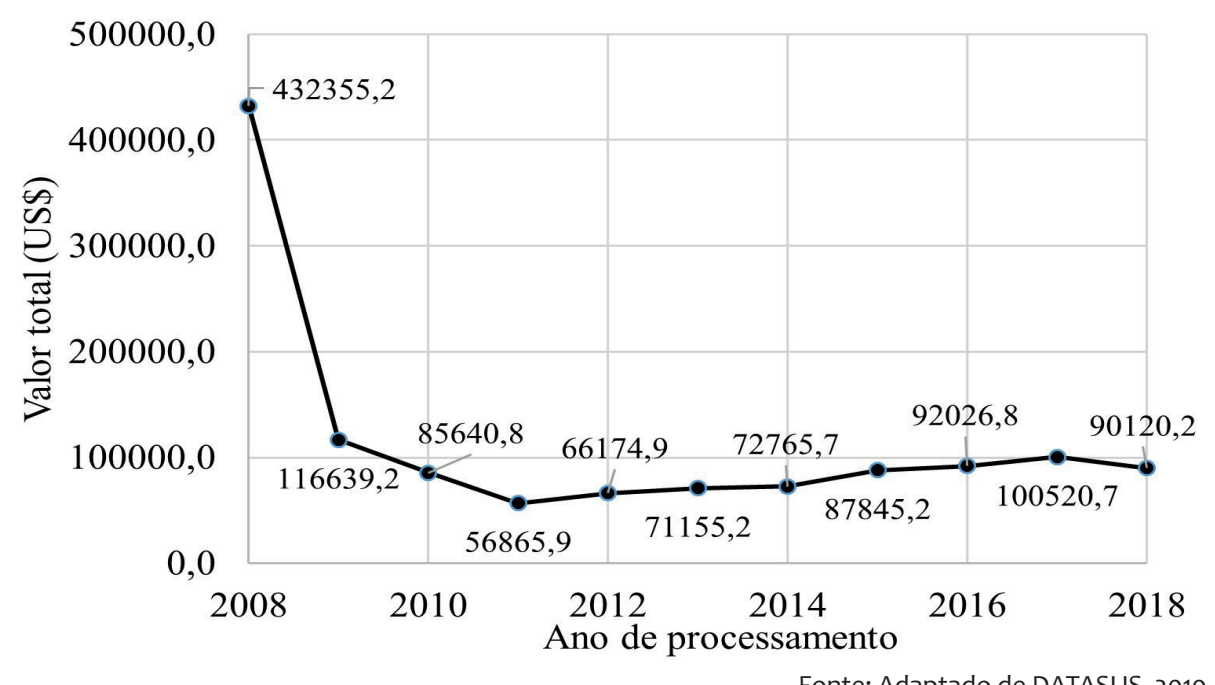

FIGURA 1: Valores totais em internações por Doença de Chagas no Nordeste brasileiro, considerando o período de 2008 a 2018

Com relação ao valor médio por internação de pessoas acometidas pela Doença de Chagas, o maior registro (US\$ 1156,0 ) ocorreu em 2008 e o menor (US\$ 405,9) em 2018, evidenciando assim um significante declínio ao longo do tempo nos gastos médios, na região do Nordeste. Ainda sobre esta variável, considerando o intervalo de 2008 a 2018, o valor registrado foi de US\$ 675,20 (Figura 2). Este é superior ao valor médio registrado em todo o Brasil, em que o gasto por internação foi equivalente a US\$545,49. ${ }^{10}$

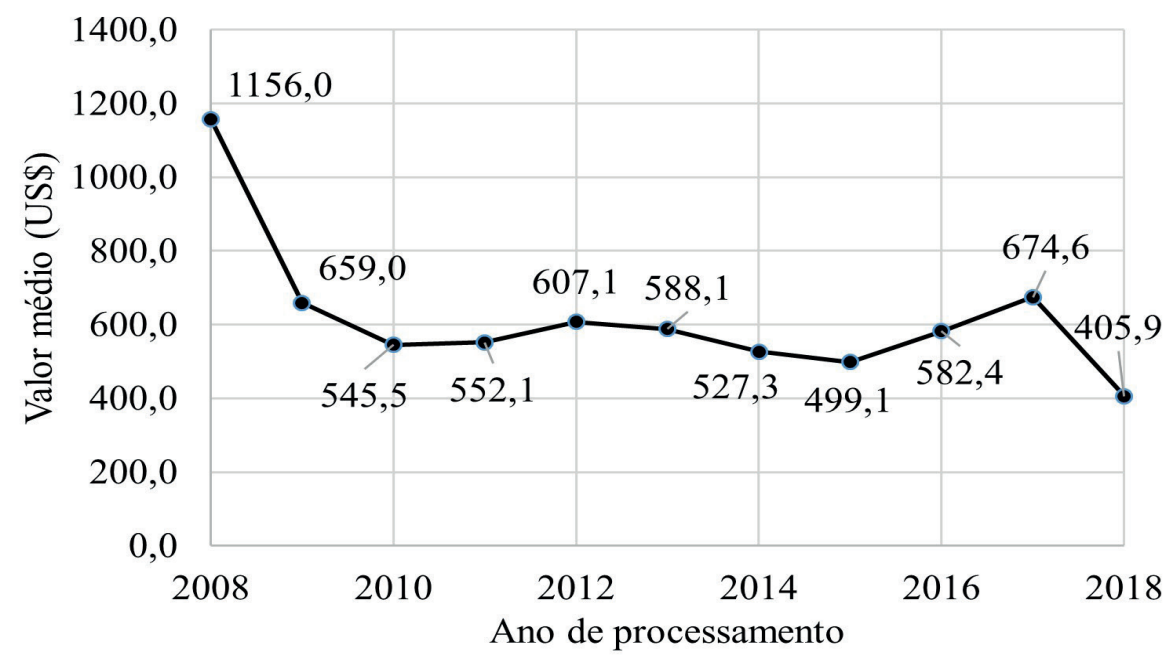

FIGURA 2: Valores médios referentes a internações por Doença de Chagas no Nordeste brasileiro, considerando o período de 2008 a 2018 
Considerando a distribuição das internações por Doença de Chagas, segundo caráter e regime de atendimento no Nordeste, durante o período estudado, pode-se observar quase $60 \%$ dos atendimentos foram de urgência e que a quantidade de internações em instituições públicas ( $\mathrm{n}=864$ ) foi quase o dobro, quando comparada com os registros de regime privado $(\mathrm{n}=455)$ (Tabela 2). Nessa perspectiva, há relatos que apontam que os pacientes com Doença de Chagas são dispendiosos ao sistema público de saúde e indesejáveis no setor privado. ${ }^{12}$

TABELA 2: Valores referentes ao caráter e regime de internações por Doença de Chagas no Nordeste brasileiro, considerando o período de 2008 a 2018

\begin{tabular}{ccccccccc}
\hline \hline \multirow{2}{*}{ Variáveis } & \multicolumn{2}{c}{ Caráter de atendimento } & & \multicolumn{3}{c}{ Regime de atendimento } & \multirow{2}{*}{ Total } \\
\cline { 2 - 3 } & Eletivo & Urgência & & Público & Privado & Ignorado & \\
\hline \multirow{2}{*}{$\mathrm{N}$} & 756 & 1128 & & 864 & 455 & 565 & 1884 \\
$\%$ & 40,1 & 59,9 & & 45,9 & 24,2 & 29,9 & 100 \\
\hline \hline
\end{tabular}

Com relação ao sexo, pode-se observar que os homens acometidos por Doença de Chagas detiveram os maiores registros em todas as variáveis estudadas (Tabela 3). A maior exposi- ção a ambientes propícios ao contato com os vetores de Doença de Chagas é um aspecto importante que deve ser considerado ao observar tal predomínio. ${ }^{13}$

TABELA 3: Valores de internações, óbitos, taxa de mortalidade hospitalar e valor médio por internação considerando a população acometida por Doença de Chagas no Nordeste brasileiro, em relação ao sexo, cor/raça e faixa etária, durante o período de 2008 a 2018

\begin{tabular}{|c|c|c|c|c|c|}
\hline Variável & Internações & Óbitos & $P$ & $\begin{array}{c}\text { Taxa de mortalidade } \\
\text { hospitalar (\%) }\end{array}$ & $\begin{array}{l}\text { Valor médio por } \\
\text { internação }\end{array}$ \\
\hline \multicolumn{6}{|l|}{ Gênero } \\
\hline $\begin{array}{c}\text { Masculino } \\
\text { Feminino } \\
\end{array}$ & $\begin{array}{r}1045(55,5 \%) \\
839(44,5 \%) \\
\end{array}$ & $\begin{array}{r}106(59,2 \%) \\
73(40,8 \%) \\
\end{array}$ & 0,353 & $\begin{array}{r}10,14 \\
8,7 \\
\end{array}$ & $\begin{array}{r}2646,04 \\
2587,22 \\
\end{array}$ \\
\hline \multicolumn{6}{|l|}{ Cor/raça } \\
\hline Branca & $95(5,1 \%)$ & $8(4,5 \%)$ & \multirow{6}{*}{ * } & 8,42 & 2131,82 \\
\hline Preta & $42(2,2 \%)$ & $4(2,2 \%)$ & & 9,52 & 1396,91 \\
\hline Parda & $626(33,2 \%)$ & $67(37,4 \%)$ & & 10,7 & 2647,35 \\
\hline Amarela & $6(0,3 \%)$ & $0(0 \%)$ & & 0 & 3620,40 \\
\hline Indígena & $0(0 \%)$ & $0(0 \%)$ & & 0 & 0 \\
\hline Não informado & $1115(59,2 \%)$ & $100(55,9 \%)$ & & 8,97 & 2686,66 \\
\hline \multicolumn{6}{|c|}{ Faixa Etária (anos) } \\
\hline Menor que 1 & $154(8,2 \%)$ & $9(5,0 \%)$ & \multirow{5}{*}{ * } & 5,17 & 1938,58 \\
\hline 1 a 9 & $152(8,0 \%)$ & $6(3,4 \%)$ & & 3,95 & 808,55 \\
\hline 10 a 19 & $71(3,8 \%)$ & $3(1,7 \%)$ & & 4,23 & 1843,53 \\
\hline 20 a 59 & $763(40,5 \%)$ & $60(33,5 \%)$ & & 7,86 & 2465,89 \\
\hline Acima de 60 & $744(39,5 \%)$ & $101(56,4 \%)$ & & 13,58 & 3362,88 \\
\hline Total & $1884(100 \%)$ & $179(100 \%)$ & & 9,5 & 2619,84 \\
\hline
\end{tabular}


Desta forma, observa-se que os homens são comumente mais expostos as condições de risco, estando mais frequentemente associado ao adoecimento por Doença de Chagas. Porém, percebe-se ainda que este perfil não é obrigatório e pode ser alterado pelas condições ambientais as quais a população, independente de sexo, esteja exposta. Tendo em vista que um estudo realizado em Porto Alegre-RS, acerca de doenças relacionadas com as más condições de saneamento (incluindo a Doença de (hagas), corroborou com os registros superiores no sexo masculino em relação aos valores médios por internação. ${ }^{14}$ Entre outros estudos nas regiões Norte e Nordeste também relataram que o sexo masculino deteve registros superiores em relação ao sexo feminino. ${ }^{13}, 15,16$ Entretanto, estudos no Sudeste apontam resultados conflitantes, em que as mulheres foram prevalentes. ${ }^{17}$

Quando se considera os dados sobre as internações por acometidos com Doença de Chagas, em relação a cor/raça evidenciam uma prevalência de indivíduos pardos em quase todas as variáveis apontadas, com um percentual de $33,2 \%$ das internações, $37,4 \%$ dos óbitos e uma taxa de mortalidade hospitalar de $10,7 \%$. O valor médio por internação foi o único quesito que a cor parda foi superada, havendo um maior registro de indivíduos da raça amarela. Vale destacar que um grande percentual de pacientes não tiveram informação sobre sua cor em termos de internações e óbitos, correspondendo a 59,2 e $55,9 \%$ do total, respectivamente. Não houve registro para a cor/raça indígena (Tabela 3).

\section{CONCLUSÕES}

De modo geral, pode-se concluir que, durante o período de 2008 a 2018, houve um declínio acentuado no número de internações com casos clínicos de
Em uma análise de casos de infecção por Doença de Chagas em Barcarena-PA, foi relatado que os indivíduos pardos e brancos foram predominantes e que não houve nenhum indivíduo da cor/raça indígena, resultados estes que corroboram com os dados aqui descritos para a região Nordeste. Por outro lado, o número de pretos e amarelos no estudo em Barcarena foram idênticos, bem como a porcentagem de registros em que a cor foi ignorada foi bem inferior, se comparada com as informações aqui descritas para o Nordeste. ${ }^{18}$

Outro trabalho, realizado na Bahia, também relatou uma predominância de indivíduos pardos em relações as demais raças. No entanto, o percentual de pretos foi superior ao de brancos, o que diverge com os resultados no Nordeste aqui relatados. O elevado número de indivíduos pertencentes a etnias nãobrancas, conforme descrito, pode ser associado a uma discrepância histórica perjorativa já que estes grupos estão associados a atividades socioeconômicas que favorecem a transmissão da doença. ${ }^{19}$

Em relação a faixa etária, observou-se que os indivíduos com Doença de Chagas com idade superior $\mathrm{a} 60$ anos detêm maiores valores de óbitos, taxa de mortalidade hospitalar e valor médio por internação, sendo superados apenas no número de internações, apesar da diferença ser pequena. Esses resultados podem estar associados ao tempo que leva para que a doença se manifeste e, quando isso ocorre, a situação clínica em muitos casos já está bem complicada. ${ }^{17}$ 
mortalidade houve muitas oscilações e em raras situações se observou uma tendência de declínio, de modo que os valores se mantiveram no mesmo nível ou até superaram o que havia sido registrado em 2008.

Com relação ao caráter e regime de atendimento de pacientes com Doença de Chagas, a maior parte das internações ocorreram no setor público e foram em caráter de urgência. Além disso, o perfil de acometidos foi, predominantemente, de indivíduos do sexo masculino, cor parda e com faixa etária superior a 60 anos.

Desse modo, é de grande relevância ampliar as informações acerca das características epidemiológicas da Doença de Chagas, a fim de conhecer a situação vigente, avaliar os aspectos determinantes para o impacto gerado pela doença e orientar as ações em saúde pública, a fim de buscar soluções viáveis para esta parasitose que afeta um grande número de pessoas no Nordeste.

\title{
CHAGAS DISEASE: HOSPITAL MORBITY PROFILE IN THE NORTHEAST BRAZILIAN REGION
}

\begin{abstract}
Chagas disease is a parasitic infection that affects millions of people worldwide, especially those living in poverty-stricken countries. Thus, the present study aims to evaluate the variables associated with hospital morbidity due to Chagas disease in Northeastern Brazil. For this, we used data collected from the SUS Hospital Information System, regarding the number of hospitalizations and deaths, hospital mortality rates, average value per hospitalization and total value of hospitalizations, regime, character of care, gender and color/race, considering the population of the Northeast, with no period from 2008 to 2018. Data for 2008 have the highest records of hospitalizations and expenses related to these rates, despite that same year and the lowest mortality rates throughout the period. In addition, from 2008 to 2018 US\$ $1,272,109.80$ were spent on hospitalizations, which is equivalent to an average value per hospitalization of US\$ 675.20. Most hospitalizations were performed in the public health system, besides being urgent. It was also found that there was a predominance of male, brown and 60 years of age or older. Thus, it can be seen that there was a considerable decrease in the number of hospitalizations over the study period, as well as the expenses associated with them, which differed with the increase in mortality that did not show any linear change over the years.
\end{abstract}

KEYWORDS: American Trypanosomiasis. Hospitalization. Hospital Services. Hospital Information Systems.

\section{REFERÊNCIAS}

1. Conners EE, Vinetz JM, Weeks JR, Brouwer KC. A global systematic review of Chagas disease prevalence among migrants. Acta Trop. 2016;156:68-78.
2. WHO. Chagas disease (American trypanosomiasis). Geneva: World Health Organization, 2019. 
1. Conners EE, Vinetz JM, Weeks JR, Brouwer KC. A global systematic review of Chagas disease prevalence among migrants. Acta Trop. 2016;156:68-78.

2. WHO. Chagas disease (American trypanosomiasis). Geneva: World Health Organization, 2019.

3. Bern C. Chagas' disease. N Engl J Med. 2015;373(5):456-66.

4. Wagner N, Jackson Y, Chappuis F, Posfay-Barbe KM. Screening and management of children at risk for Chagas disease in nonendemic areas. Pediatr Infect Dis J. 2016;35(3):335-7.

5. Martin-Escolano R, Molina-Carreno D, Delgado-Pinar E, Martin-Montes A, Clares MP, Medina-Carmona $\mathrm{E}$, et al. New polyamine drugs as more effective antichagas agents than benznidazole in both the acute and chronic phases. Eur J Med Chem. 2019;164:27-46.

6. Stanaway JD, Roth G. The burden of Chagas disease: estimates and challenges. Glob Heart. 2015;10(3):139-44.

7. IBGE. Sistema IBGE de Recuperação Automática - SIDRA 2019 [Available from: https://sidra. ibge.gov.br/home/pnadcm.

8. Santos VRC, Meis J, Savino W, Andrade JAA, Vieira JRS, Coura JR, et al. Acute Chagas disease in the state of Pará, Amazon Region: is it increasing? Mem Inst Oswaldo Cruz. 2018;113(5).

9. Schofield CJ, Jannin J, Salvatella R. The future of Chagas disease control. Trends in Parasitology. 2006;22(12):583-8.

10. Silva AP, Dantas BB. Morbimortalidade hospitalar decorrente de doença de Chagas no Brasil, de 2008 a 2018. Editora Realize [In- ternet]. 2019; 1:[1-10 pp.]. Available from: https://editorarealize.com.br/revistas/conapesc/ trabalhos/TRABALHO_EV126_MD1_SA10_ ID1204_30062019224147.pdf.

11. Lee BY, Bacon KM, Bottazzi ME, Hotez PJ. Global economic burden of Chagas disease: a computational simulation model. Lancet Infect Dis. 2013;13(4):342-8.

12. Bozelli CE, Araújo SM, Guilherme ALF, Gomes ML. Perfil clínico-epidemiológico de pacientes com doença de Chagas no Hospital Universitário de Maringá, Paraná, Brasil. Cad Saúde Pública. 2006;22(5):1027-34.

13. Teixeira RB, Oliveira SMC. Perfil de pacientes portadores de Doença de Chagas em rio Branco, Acre, Brasil. Rev Soc Bras Clín Méd. 2015;13(4).

14. Siqueira MS, Rosa RS, Bordin R, Nugem RC. Internações por doenças relacionadas ao saneamento ambiental inadequado na rede pública de saúde da região metropolitana de Porto Alegre, Rio Grande do Sul, 2010-2014. Epidemiol Serv Saúde. 2017;26:795-806.

15. Santana MP, Souza-Santos R, Almeida AS. Prevalência da doença de Chagas entre doadores de sangue do Estado do Piauí, Brasil, no período de 2004 a 2013. Cad Saúde Pública. 2018;34:e00123716.

16. Vieira JFPN. Doença de Chagas no Piauí distribuição geográfica dos óbitos de 2003 a 2013 e identificação de vetores em comunidades rurais de São João do Piauí [Dissertação]. Teresina: Instituto Oswaldo Cruz; 2017.

17. Guariento ME, Carrijo CM, Almeida EA, Magna LA. Perfil clínico de idosos portadores de doença de Chagas atendidos em serviço de referência. Rev Bras Clin Med. 2011;9(1):20-4. 
18. Júnior S, Da Silva A, Palácios VRCM, Miranda CS, Costa RJF, Catete CP, et al. Análise espaço-temporal da doença de Chagas e seus fatores de risco ambientais e demográficos no município de Barcarena, Pará, Brasil. Rev Bras Epidemiol. 2017;20:742-55.
19.Braga JCV, Reis F, Aras R, Costa ND, Bastos C, Silva $R$, et al. Aspectos clínicos e terapêuticos da insuficiência cardíaca por doença de Chagas. Arq Bras Cardiol. 2006;86(4):297-302. 